\title{
La Voce dei Pazienti Scelte alimentari e di lavoro. Rene policistico e vita quotidiana
}

\author{
Essere affetti da una patologia come il rene policistico comporta riflessioni spesso anche drammatiche sulle grandi \\ scelte della vita (prima fra tutte, quella di avere o non avere figli). \\ Ma altrettanto importanti sono le decisioni \\ - solo apparentemente meno importanti - relative alla vita quotidiana. \\ Sulla pagina Facebook di AIRP, si è recentemente dibattuto sugli stili di alimentazione più adatti a chi soffre \\ di rene policistico e sul tema dell'invalidità in relazione al mondo del lavoro. Entrambi gli argomenti hanno \\ generato fitti scambi di esperienze e di opinioni, con un'intensa partecipazione di voci diverse...
}

S.V.: Salve a tutti, è la prima volta che scrivo. Anche $i$ miei reni sono pieni di cisti, ma per fortuna ancora funzionano. Vorrei fare una domanda: che effetto può avere, nella nostra condizione, adottare una dieta vegana?

R.P.: Ottima scelta, le proteine animali fanno lavorare di più i reni, quindi meglio non sovraccaricarli, meglio proteine ricavate dai legumi.

S.V.: È quello che ho pensato anch'io, anche se la dietologa dell'ospedale non è d'accordo...

R.P.: Salve, volevo dire che la dieta vegana è un'ottima scelta: i reni si affaticano meno.

E lavorano meglio. Proprio oggi il mio medico mi diceva che l'ha consigliata anche ad altri pazienti. Io sono il risultato: creatinina scesa. Lui era soddisfatto e io con lui.

M.S.: Ma le carni bianche si possono mangiare? Oppure sono sconsigliate anche quelle? E le uova?

L.S.: Ma non siete in dialisi? Perché a me hanno detto di mangiare molte proteine...

J.T.: Pochi giorni fa sono andata dalla dietologa proprio per avere dei chiarimenti. Io sono alta 1.73 , peso $73 \mathrm{~kg}$ e ho $2 \mathrm{di}$ creatinina; in base a questi dati, ha calcolato che posso mangiare 250 grammi di carboidrati al giorno tra fette biscottate, pane e pasta, e 125 grammi a scelta tra carne, pesce, formaggi freschi, due uova, prosciutto, oppure 80 grammi di legumi sempre al giorno. Ho chiesto riguardo a caffè, tè, cioccolata, latticini per via del lattosio. Mi ha solo detto di non esagerare, ma io già non bevo più di un caffè e prendo solo un tè al giorno... Però sappiamo tutti che le proteine animali affaticano $\mathrm{i}$ reni e anche il lattosio; quindi, io continuo a evitare proteine animali. So che a breve uscirà un libro dell'Airp proprio sull'alimentazione, così ci chiariremo ogni dubbio.
R.P.: Buon giorno a tutti. Sostengo da sempre che la dieta è importante. Mia sorella ha la malattia policistica, per sua scelta è stata sempre vegetariana, ha 57 anni è sta bene. È seguita da un medico ayurvedico (i medici normali non ne capiscono niente). Adesso, anch'io sto seguendo la sua dieta. Ho letto su una rivista che per i nefropatici consigliano la dieta vegana. I risultati li vedo dai miei esami. Bevo caffè decaffeinato, non bevo tè, vivo tranquillamente senza proteine animali. L'unica cosa che mi manca sono i formaggi. Adesso finché sto bene non cambio alimentazione. Mi spiace: se 1'avessi saputo prima avrei difeso i miei reni da subito...

M.S.: Giusto, se l'avessi saputo prima, anch'io avrei difeso meglio i miei reni. Da quando ho sostituito le proteine animali con quelle vegetali, la creatinina si è abbassata.

R.P.: Evvai! Provare non costa niente. Anzi, se possiamo evitare la dialisi, penso proprio che ne valga la pena!

S.V.: Io ho iniziato questo "percorso" leggendo un libro che parla di un collegamento tra alimentazione e malattia, vi garantisco che è molto interessante: The China studi del Dr. Campbell. Non ci sono riferimenti al rene policistico, ma ho pensato che - considerata la situazione - già evitare altre patologie era un gran passo. Per ora mi trovo bene e le ultime analisi erano perfette, vedremo le prossime... nel frattempo grazie a tutti.

R.V.: Dove lo hai trovato questo libro?

S.V.: Nel reparto libreria di un supermercato Pam... comunque non dovrebbe essere difficile trovarlo, c'è anche su Facebook.

M.G.: Tieni conto che la vegana è ricca di proteine. E anche di queste proteine, seppure vegetali, tipo fagioli ecc., bisognerebbe mangiarne solo di rado! 
R.P.: Condivido: io li mangio una volta la settimana.

J.T.: Scusa, mi puoi fare un esempio del tuo menu giornaliero? Carne no, formaggi no, uova e fagioli una volta la settimana... mangi solo pasta e verdura? Mi fai un favore perché io cominciò a essere povera di idee... Grazie.

C.C.: Ciao. Volevo chiedere a R.P. - dato che sono anni che penso di seguire una dieta vegana e me l'hanno sempre sconsigliata, perché il medico dice che si va incontro a malnutrizionetu avevi la crea alta e con la dieta ti è scesa?

R.P.: Sì, avevo la creatinina a 2 e altri valori alterati. Da quando seguo questa dieta, la creatinina é scesa a 1 e la velocità di filtrazione del sangue nei reni è aumentata. Non sono malnutrita, non ho anemia, il calcio lo prendo dagli integratori.

C.C.: Già, sono i medici che dicono che è un mal nutrirsi. Sarei felice di avere indicazioni, se puoi, dove trovare magari tabelle, ricette ecc. Vorrei provarci. Dopo quanto tempo dall'inizio della dieta hai rifatto le analisi?

R.P.: Almeno 6 mesi la prima volta. In realtà, non c'è una vera dieta con ricette: bisogna semplicemente togliere la carne bianca e rossa, salumi, formaggi (al supermercato trovi la carne vegetale) e poi alterni melanzane, zucchine, cavolfiore, peperoni, verdura, frutta, legumi, frutta secca... Il pesce una volta al mese e anche l'uovo. L'unico formaggio che mangio è la mozzarella nella pizza.

\section{Mettere o non mettere l'invalidità nel curriculum?}

F.S.: Salve a tutti. Sto preparando il curriculum vitae da inviare a diverse aziende private. Avendo un'invalidità civile al 70\%, devo farlo presente nel curriculum e nella lettera di presentazione?

E.S.: Se sei già iscritta alle categorie protette sì. $\mathrm{O}$ almeno io l'ho indicato nel cv.

M.S.: Sì, ti devi iscrivere presso il Centro per l'Impiego della tua città e poi mandi il curriculum precisando: "Appartenente alle categorie protette (70\%). Collocabile ai sensi della Legge 68/1999". Per chi ti assume, ci sono agevolazioni fiscali molto favorevoli e il datore di lavoro ha l'obbligo di non farti svolgere lavori che non sono idonei per la tua patologia (sollevare pesi, stare sempre in piedi o sempre seduta, dipende dai problemi che hai).

C.N.: Sì, ma che possibilità ci sono di trovare lavoro? Io è da tre anni che sono iscritto a Varese e non si trova niente...

L.G.: Se sei iscritta all'ufficio di collocamento mirato, ti possono assumere come categoria protetta, loro ci guadagnano e tu pure: hai il posto fisso, anche se magari dopo un anno di prova, ma poi non ti cacciano più.
M.S.: Sì, certo, iscritta nella lista delle categorie protette nel collocamento mirato. Io sono iscritta da quasi cinque anni e non ho trovato niente! Ormai le possibilità sono veramente poche, anzi quasi nulle! A meno che non abiti in una grande città e allora forse qualche possibilità in più potresti averla.

L.G.: Bisogna collegarsi al sito della Provincia e curare quando escono i concorsi per le categorie protette, seguire la trafila della domanda al Centro per l'Impiego e poi entrare in graduatoria. Io all'ultimo concorso per il Tribunale sono arrivata 24esima, però bisogna sostenere una prova scritta e non penso di avere molte possibilità di arrivare prima. A meno che i primi 23 non sappiano stilare una lettera in word dietro dettatura a una certa velocità. Ormai anche i posti nei Comuni e i posti statali sono rari, dato che non hanno più soldi neanche per mantenere i servizi che offrono attualmente. Quindi noi, oltre ad avere il disagio della malattia, dobbiamo rimanere anche senza lavoro... ma se una ditta chiude, non sono obbligati a trovarti un altro impiego, se sei invalido?

G.E.: Per quanto io sappia, scatta l'assunzione obbligatoria di categorie protette solo per le aziende che hanno più di quindici dipendenti. In tal caso, le aziende saranno agevolate, altrimenti nel privato non si favoriscono assunzione con agevolazione. Ad ogni modo, se si è invalidi al $70 \%$ non si può omettere la cosa quando si deve essere assunti. Inoltre, per rientrare nella lista è necessario fare domanda all'INPS e poi successivamente al centro per l'impiego.

R.P.: Devi essere iscritta alla Camera del Lavoro, dove verrai valutata per capire che tipo di lavoro puoi fare.

R.G.: Te lo dico da imprenditore: se devi inviare dei curricula, non lo scrivere. Fallo presente solo agli uffici di collocamento. Il perché mi sembra ovvio...

L.G.: Ma se poi alcune mansioni non le puoi fare, come nel mio caso? O lo collochi in ufficio come è successo a me dopo aver chiamato l'Asl, o tu licenzieresti l'operaio?

F.S.: Grazie a tutti, mi avete risposto veramente in tanti... comunque, dato che sono in grado di svolgere tutte le mansioni che richiede il lavoro del mio caso, per il momento lo ometto.

R.G.: Secondo me è meglio. Io, se avessi bisogno di personale - scusate la franchezza - prenderei una persona sana, possibilmente maschio, sui 30 anni, e chiamerei i precedenti datori di lavoro per saperne di più. Non dimenticate di inserire se avete l'auto e/o figli. Chi ha dei figli, io lo avvantaggio, perché significa che ha bisogno di lavorare.

G.E.: Comprendo ciò che dici, ma trovo bizzarro che prima esigi determinati requisiti dai tuoi dipendenti e poi a una ragazza che già perché donna non assumeresti, per giunta con invalidità, consigli di nascondere la sua invalidità perché rischia di non essere assunta. 
R.G.: Il mio è solo un consiglio. Comunque nel mio piccolo ho assunto cinque donne su cinque posti e tutte in età fertile. Ripeto. Il mio è un consiglio ma è anche la dura realtà.

M.S.: Ma perché nascondere l'invalidità? Assumendo una persona con invalidità, il datore di lavoro ha delle agevolazioni fiscali. E poi, rischio di essere collocata a compiti che non riuscirei a svolgere in quanto invalida. Sino ad ora ho solo ricevuto offerte per lavori di fatica che non riesco più a fare, come avrei dovuto comportarmi? Nascondere la mia invalidità, farmi assumere e poi? Essere considerata una cretina per non aver segnalato un'invalidità così importante?

F.S.: Non preoccuparti per questo, sto inviando il curriculum a vari laboratori di analisi privati in quanto sono un tecnico di laboratorio, non mi manderanno in miniera, al massimo dovrò spostare dei bidoni di reagenti! Sono una donna in età fertile, di sinistra e agnostica... spero ci sia un posto di lavoro anche per me. La mia invalidità non mi crea alcun problema per ora, sto bene!

\section{Una nuova paziente viene accolta nel gruppo}

G.C.: Buonasera a tutti! Ho scoperto solo poche settimane fa di essere affetta da rene policistico, e oggi sono stata per la prima volta in vita mia da un nefrologo. Anche se per ora si tratta di una notizia un po'difficile da digerire, spero che con il tempo avrò sempre più chiara la mia situazione e capirò come devo comportarmi per star bene il più possibile... Intanto, grazie per aver accettato la mia richiesta, è importante un confronto con chi già conosce questo disturbo e ci convive!

K.T.: Ciao io sono K. Quattro anni fa abbiamo scoperto la malattia a mia figlia di soli 12 anni. Ti capisco, perché anche noi siamo sprofondati in un pozzo nero, ma da lì bisogna risollevarsi per il suo bene, per non farla vivere come malata, abbiamo imparato a vivere un giorno alla volta, si può fare solo questo, e lo si deve fare sempre con il sorriso...

A.C.: Ciao G.! Noi famiglia abbiamo ereditato dalla famiglia questa patologia da cui anch'io da anni sono affetto. Posso dirti che è importante condurre una vita serena e regolare, come ogni essere umano, e fare annualmente un'ecografia reni/fegato per controllare lo stato e l'evoluzione di queste cisti liquide. Stai tranquilla cara G. Un forte abbraccio!

G.C.: Grazie! Anche la dottoressa mi ha detto di stare tranquilla. Ci proverò, promesso. Un abbraccio grande!

F.G.: Ciao G., benvenuta nel gruppo. Io sono moglie e mamma di tre bellissimi ragazzi tutti policistici. Andiamo avanti. Ciao.

J.T.: G., stai serena. Dovrebbe uscire presto il Tolvaptan, che impedirebbe la crescita delle cisti. Io ci convivo da trent'anni e ho 2 di creatinina. L'evoluzione potrebbe essere più lenta di quanto si pensi, quindi... limita le proteine animali e soprattutto VIVI!

G.C.: Agli ordini!! Di mio sono una persona ottimista, quindi non mi lascerò abbattere... Incrociamo le dita per il Tolvaptan. Nell'attesa, anche io cercherò di vivere il più serenamente possibile questa malattia. Solo, con un pizzico di consapevolezza in più! Lo userò come sprone per condurre una vita sana. Per esempio, mi son finalmente decisa a ricominciare a nuotare!! Ciao a tutti. 